\title{
Electronic Properties of Tin and Bismuth from Angular Correlation of Annihilation Photons
}

\author{
Mogensen, O.E.; Trumpy, Georg
}

Published in:

PHYSICAL REVIEW

Link to article, DOI:

10.1103/PhysRev.188.639

Publication date:

1969

Document Version

Publisher's PDF, also known as Version of record

Link back to DTU Orbit

Citation (APA):

Mogensen, O. E., \& Trumpy, G. (1969). Electronic Properties of Tin and Bismuth from Angular Correlation of Annihilation Photons. PHYSICAL REVIEW, 188(2), 639-644. https://doi.org/10.1103/PhysRev.188.639

\section{General rights}

Copyright and moral rights for the publications made accessible in the public portal are retained by the authors and/or other copyright owners and it is a condition of accessing publications that users recognise and abide by the legal requirements associated with these rights.

- Users may download and print one copy of any publication from the public portal for the purpose of private study or research.

- You may not further distribute the material or use it for any profit-making activity or commercial gain

- You may freely distribute the URL identifying the publication in the public portal

If you believe that this document breaches copyright please contact us providing details, and we will remove access to the work immediately and investigate your claim. 


\title{
Electronic Properties of Tin and Bismuth from Angular Correlation of Annihilation Photons
}

\author{
O. E. Mogensen* and G. Trumpy \\ Laboratory of Applied Physics II, Technical University of Denmark, Lyngby, Denmark
}

(Received 3 February 1969; revised manuscript received 25 July 1969)

\begin{abstract}
A linear slit setup has been used to obtain results of angular-correlation measurements in (a) tin single crystals in three orientations: [001], [100], and [110], (b) bismuth single crystals in four orientations: [111], [100], [1i0], and [2i 1$]$, (c) solid and liquid tin and bismuth, and (d) deformed bismuth. For both metals, the single-crystal angular-correlation curves lie near to the free-electron parabola. The tin curves show more anisotropy than the bismuth curves. An important result is the clear anisotropy found in the high-momentum part of the curves-the tails-for both metals. Little agreement is found between simple theoretical models and these results. A calculation of the curves based on the one-electron theory of valence electrons in metals would be highly desirable. The peaks of the liquid-metal curves are narrower than the peaks of the polycrystalline curves. The tails of the liquid-metal curves are smaller and of another form than the tails of polycrystalline curves; no Gaussian with only one adjustable constant factor can give a fit to both tails. No useful method for interpreting liquid-metal angular-correlation curves seems to exist. Two deformed bismuth samples gave angular-correlation curves nearly equal to the polycrystalline curves.
\end{abstract}

\section{INTRODUCTION}

$\mathrm{T}$ HE experimental study of the photons emitted when positrons annihilate with electrons in a metal has been found ${ }^{1}$ to be a useful method for obtaining information about the properties of the electrons. At present there is a growing interest in theoretical and experimental studies of the electrons away from the Fermi surface. It is a remarkable fact that most of the measurements which can check the one-electron band theory and the electron-gas many-body theory involve only electrons on the Fermi surface. Positron annihilation experiments constitute one of the very few sources of information about valence electrons within the Fermi surface. Other competing techniques involve measurements of soft $x$-ray emission, ${ }^{2}$ optical properties, photo emission, and Compton scattering. ${ }^{3,4}$ The present paper contains the results of linear slit measurements of the angular correlation of annihilation photons in single crystals of tin and bismuth. We also report on angularcorrelation experiments in liquid tin and bismuth and in deformed bismuth. We have been particularly careful to eliminate different types of background countings and we have thus been able to cover an unusually large angle interval. This allows a detailed examination of the tails of the angular-correlation curves. We believe that it should be possible to use the data of this report as a test of the applicability of band-structure wave functions for electrons within the Fermi surface in tin and bismuth.

* Present address: Institute of Chemical Physics, Academy of Sciences, Moscow, USSR.

${ }_{1}$ Proceedings of the Positron Annihilation Conference, Detroit, 1965, edited by A. T. Stewart and L. O. Roellig (Academic Press Inc., New York, 1965).

${ }^{2}$ Soft X-ray Band Spectra and the Electronic Structure of Metals and Materials, edited by D. J. Fabian (Academic Press Inc., New York, 1968).

${ }^{3}$ W. C. Phillips and R. J. Weiss, Phys. Rev. 171, 790 (1968).

${ }^{4}$ W. C. Phillips and R. J. Weiss, Phys. Rev. 182, 923 (1969).

\section{THEORY}

In a metal the positron and electron can be represented by the Bloch functions

$$
\begin{aligned}
& \psi_{\mathrm{p}} \propto u_{\mathrm{p}}(r) e^{i \mathrm{p} \cdot \mathrm{r}}, \\
& \psi_{\mathrm{q}} \propto u_{\mathrm{q}}(\mathbf{r}) e^{i \mathrm{q} \cdot \mathrm{r}} .
\end{aligned}
$$

The usual linear slit angular-correlation setup ${ }^{1}$ determines the distribution of a component $k_{z}$ of the total photon momentum $\mathbf{k}$. By use of the expansion

$$
u_{\mathrm{p}}(\mathbf{r}) u_{\mathrm{q}}(\mathbf{r})=\sum_{\mathbf{g}} a_{\mathrm{g}} e^{i \mathbf{g} \cdot \mathbf{r}}
$$

where the sum is over all reciprocal-lattice vectors $\mathbf{g}$, the measured intensity is, in the independent-particle model,

$$
\begin{aligned}
d w_{3} \propto \int d k_{x} \int d k_{y} \int \sum_{|\mathbf{q}| \leq q F} \sum_{\mathbf{g}} \mid & \left.a_{\mathbf{g}}(\mathbf{q})\right|^{2} \\
& \times \delta(\mathbf{g}-\mathbf{k}+\mathbf{g}) d k_{z} d \Omega_{1},
\end{aligned}
$$

where $q_{F}$ is the Fermi momentum and $d \Omega_{1}$ is the spaceangle element for one of the photons. Here it has been assumed that the positrons are thermalized or nearly thermalized $^{5}$ so that $\mathbf{p}=0$. Most of the annihilations occur with the valence electrons. The angular-correlation curve in the free-particle model is a parabola, which is a good approximation to the central part of the curve - the peak-for all orthogonal-plane-wave (OPW) metals. The difference between the free-electron parabola and the valence-electron peak arises because (a) the Fermi surface is different from a sphere, and (b) the wave-function products also contain Fourier components $a_{\mathfrak{g}}(\mathbf{q})$ for $\mathbf{g} \neq 0$. If we assume that $a_{\mathfrak{g}}(\mathbf{q})=0$ for $\mathbf{g} \neq 0$ and $a_{0}(\mathbf{q})$ is independent of $\mathbf{q}$, the angularcorrelation curve is proportional to the cross-sectional area of the volume within the Fermi surface in the ex-

${ }^{5}$ J. P. Carbotte and H. L. Arora, Can. J. Phys. 45, 387 (1967). 


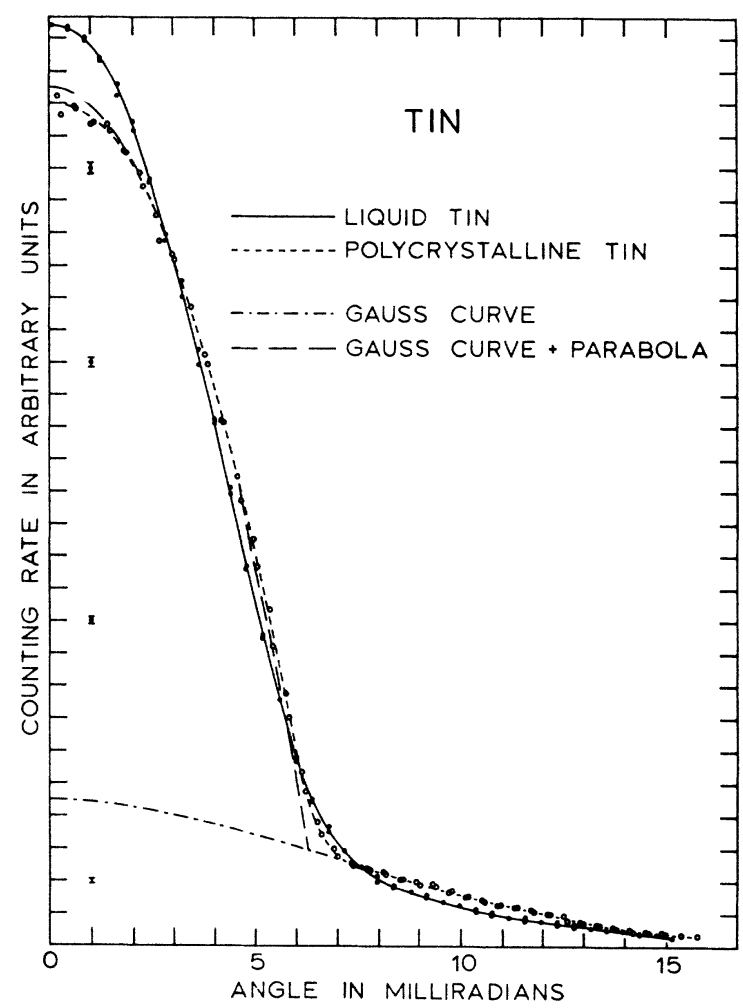

Fig. 1. Angular correlation of annihilation photons in liquid and polycrystalline tin. The two curves are normalized to equal areas.

tended zone scheme. This approximation is often used as a first qualitative model..$^{6,7}$

In the independent-particle model only part of the Coulomb interaction between the particles is taken into account. A more complete electron-gas calculation of the annihilation probability $w(\mathbf{q})$ as a function of the electron momentum $\mathbf{q}$ can be described by use of an enhancement factor $\epsilon(\mathbf{q})=w(\mathbf{q}) / w_{0}$, where $w_{0}$ is the free-electron annihilation rate. Although there seems to be some ambiguities between the different treatments, ${ }^{8-10}$ they all indicate that $\boldsymbol{\epsilon}(\mathbf{q})$ depends rather weakly on $\mathbf{q}$, especially for high-density metals like tin and bismuth. This fact and experimental results show that the independent-particle model is a good approximation for calculation of angular-correlation curves.

\section{APPARATUS}

Two-photon angular-correlation curves were obtained with a conventional linear slit setup. ${ }^{1}$ The detectors were 4-in.-diam. 1-in.-high $\mathrm{NaI}(\mathrm{Tl})$ crystals placed

${ }^{6}$ S. Berko, S. Cushner, and J. C. Erskine, Phys. Letters 27A, 668 (1968).

7.J. C. Erskine and J. D. McGervey, Phys. Rev. 151, 615 (1966)

${ }^{8}$.J. P. Carbotte, Phys. Rev. 155, 197 (1967).

9 J. Arponen and P. Jauho, Phys. Rev. 167, 239 (1968).

${ }_{10}$ A. Hatano, H. Kanazawa, and Y. Mizuno, Progr. Theoret.

Phys. (Kyoto) 34, 875 (1965). behind 1-mm-wide horizontal lead slits at a distance of about $2 \mathrm{~m}$ from the sample and the source. The irradiated plane surface of the sample was exactly horizontal. When the correlation angle $\theta=0$, the plane through the slits had an inclination angle of $20 \mathrm{mrad}$ with respect to the sample surface, so that the stationary counter could see only the face of the sample and the other could only see its side. The source was $10-\mathrm{mCi} \mathrm{Na}{ }^{22} \mathrm{Cl}$. The time spent counting in each position was $30 \mathrm{~min}$ and the number of positions were 78 for all the measured curves. Since many sweeps were necessary to obtain a single curve, effects due to drift in the electronics were thus minimized. The pulse-handling electronics was a conventional fast-slow coincidence system with a fast coincidence resolving time of $40 \mathrm{nsec}$.

The measured curve differs from the distribution of a component of the total photon momentum by a systematic error, which is caused by the finite effective slit length, being $10 \mathrm{~cm}$. This error implies that the top of the peak of an angular-correlation curve is reduced by about $1.5 \%$ relative to the lower part of the peak. The data shown in this paper are not corrected for this error. A calculation of the finite slit length errors has been published by Arifov et al. ${ }^{11}$ This treatment overestimates the errors, because only point samples are treated. It can be shown ${ }^{12,13}$ that a point sample gives larger errors than a sample whose length in the direction of the slit is comparable to the width of the distribution at the slits.

The resolution of the apparatus is determined by the finite slit widths corresponding to a broadening of 0.5 -mrad half-width, and by the fact that samples were tilted slightly relative to the plane containing the slits. The effective resolution can be approximated by a normal error curve with a half-width of about 0.65 mrad. The thermal motion of the positrons corresponds to a broadening with a half-width of $0.53 \mathrm{mrad}$ at $300^{\circ} \mathrm{K}$.

Annihilation in atmospheric air surrounding the sample was found to cause asymmetries in the measured curves; especially, the top of the parabolic peak was distorted. Hence, all the data shown here were taken with the samples surrounded with a hydrogen atmosphere.

\section{MEASUREMENTS}

In the studies of liquid metals, the sample was contained in a shallow stainless-steel box placed just below the positron source. The upper surface of the sample was $1 \mathrm{~cm}$ broad and $4 \mathrm{~cm}$ long; the longer side being parallel to the detector slits. The walls of the stainlesssteel box contributed about $2 \%$ of the total annihila-

11 P. U. Arifov, V. I. Goldanskii, and Yu. S. Sayasov, Fiz. Tverd. Tela 6, 3118 (1964) [English transl.: Soviet Phys.-Solid State 6, 2484 (1965)].

12 O. Mogensen, thesis, Technical University of Denmark, 1968 (unpublished).

${ }_{13} \mathrm{O}$. Mogensen (unpublished). 
tions. The temperature was automatically kept constant about $15^{\circ} \mathrm{C}$ above the melting point with an uncertainty of $\pm 2{ }^{\circ} \mathrm{C}$. A surface layer probably consisting of oxides was present, and attempts to avoid its formation by use of flowing hydrogen were only partly successful. The contribution of this layer to the measured curves is probably negligible, as the layer apparently is much thinner than a typical penetration depth of a positron.

The polycrystalline metal samples were made by letting the liquid metal solidify in the container. The upper surface was then ground flat, and the samples annealed at a temperature just below the melting point for some hours. A polycrystalline bismuth curve also was taken by use of a sample which was treated in the same way as the single-crystal samples and afterwards annealed. The two curves were equal within the statistical counting fluctuations. Strongly deformed samples were made simply by hammering annealed polycrystalline pieces of bismuth having roughly the same size as the single-crystal samples. We used the highest degree of deformation which could be obtained without cleaving the samples.

The tin single-crystal samples were spark-cut from an 18-mm-diam., 20-mm-long, 99.99\%-pure single crystal, furnished by Metals Research, England. A 0.1-mm layer of the surface was removed by electropolishing,

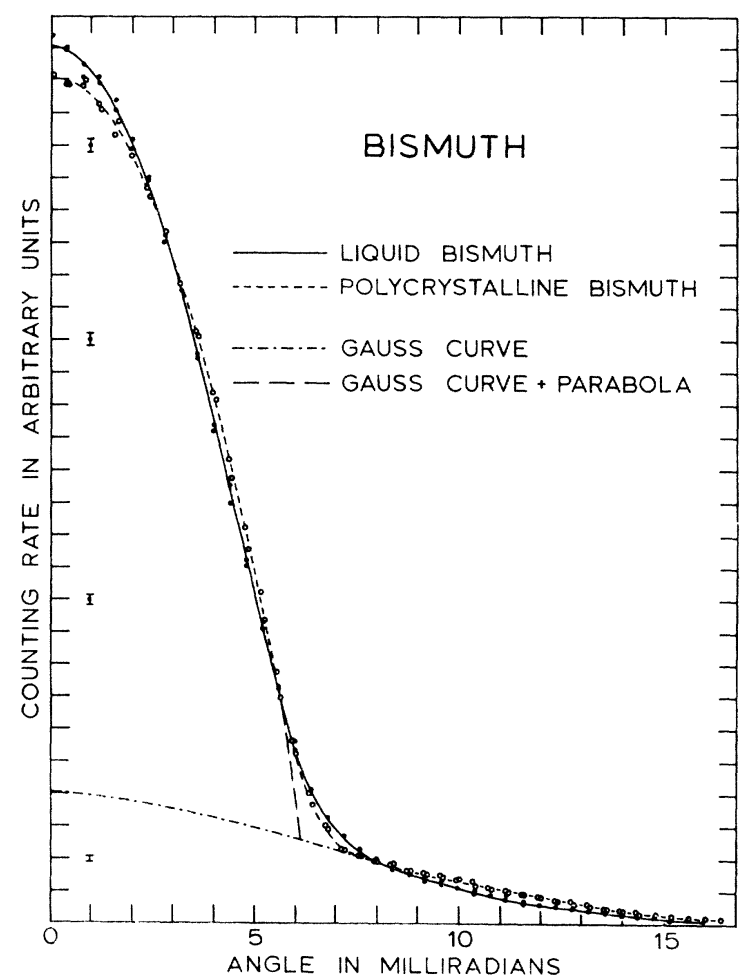

FIG. 2. Angular correlation of annihilation photons in liquid and polycrystalline bismuth. The two curves are normalized to equal areas.

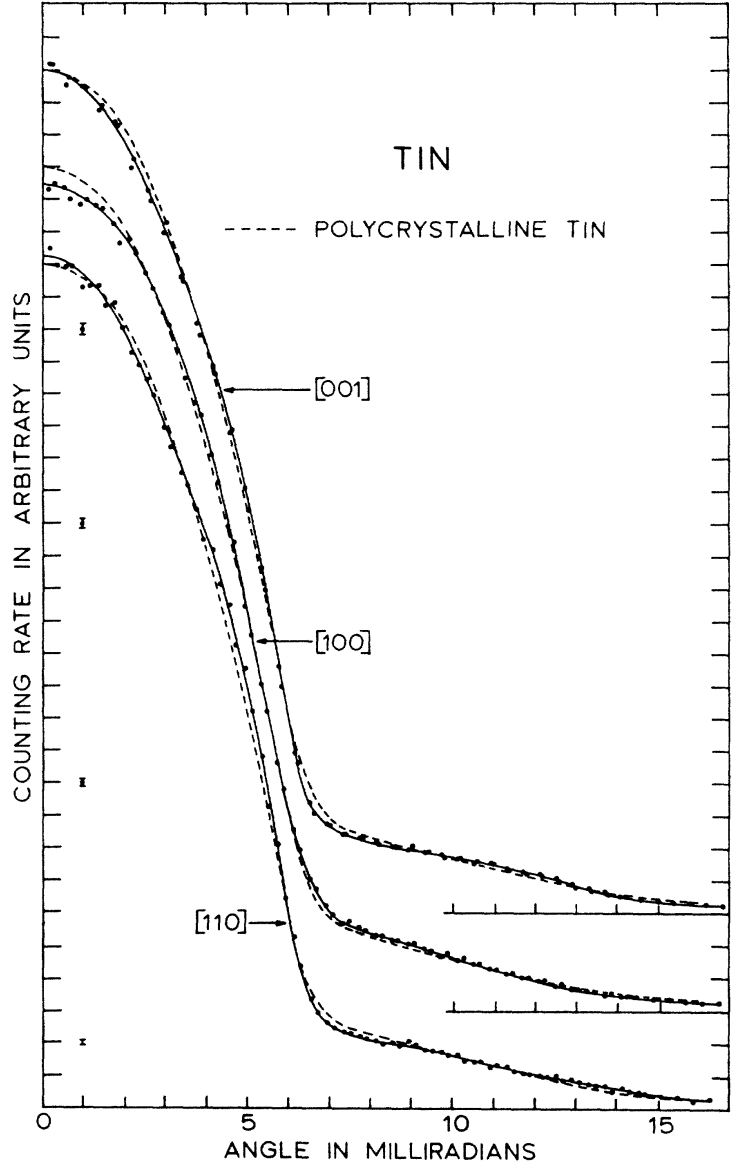

FIG. 3. Angular correlation of annihilation photons in single crystals of tin. The curves are normalized to the same area as the polycrystalline sample curve.

and the structural perfection was checked by etching. The angular-correlation curves were measured for the $[001],[100]$, and $[110]$ directions.

The bismuth single-crystal samples were spark-cut from a 25-mm-diam., 25-mm-long, 99.99\%-pure single crystal, also furnished by Metals Research. A 0.1-mm surface layer was removed by use of different abrasive powders, the samples were then etched in a nitric-acid solution, and the structural perfection was checked by etching. We measured the angular-correlation curve for the $[111],[100],[1 \overline{1} 0]$, and [2/1] directions.

The upper surface of the samples was approximately $20 \times 15 \mathrm{~mm}$, the longer side being parallel to the slit. The number of counts in the top of the curve were between 14500 and 17700 for the tin case and between 12400 and 18000 for the bismuth case. Typical maximum coincidence counting rates were about 95 per minute for tin and about 54 per minute for bismuth.

\section{RESULTS}

All the measured angular-correlation curves were obtained at intervals of $0.399 \mathrm{mrad}$ between the posi- 


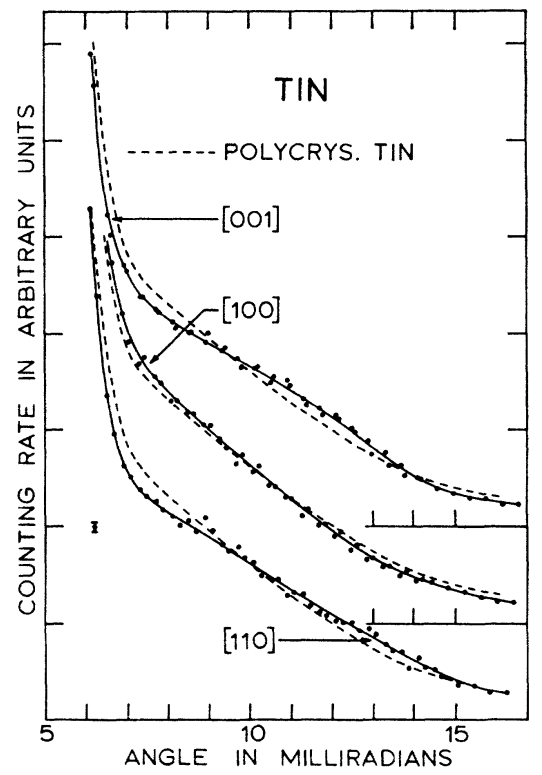

FIG. 4. Larger angle parts of the angular-correlation curves for tin single crystals.

tions of the movable slit. The curves were corrected for a background of about 14 coincident counts per hour. Figures 1-6 show the liquid-metal and single-crystal curves normalized to the same area as the polycrystalline sample curves. For comparison the latter are included in all the figures. The uncertainties shown in the figures are the statistical counting fluctuations; in Figs. 1 and 2 for the liquid-metal curves and in Figs. 3-6 for the innermost single-crystal curves.

Angular-correlation curves for polycrystalline tin and bismuth have been measured several times before..$^{1,14-16}$ Figure 2 illustrates that a free-electron parabola plus a Gaussian curve fitted to the tail is a very good approximation to the polycrystalline bismuth curve. The same approximation is not so good when used in the tin case, where the peak is broader than the free-electron parabola (see Fig. 1). Angular-correlation curves for nearly all liquid metals with low melting points have been published before.,15,16 The present tin curve has better resolution and larger angles than previous results. ${ }^{15}$ Within the uncertainties, the bismuth curve is consistent with the results by West $e t a .^{16,17} \mathrm{We}$ also studied the angular correlation for two different samples of bismuth strongly deformed in an arbitrary way. One of the curves was equal to the nondeformed bismuth curve shown in Fig. 2. The other one was slightly

${ }^{14}$ I. K. MacKenzie, G. F. O. Langstroth, B. T. A. McKee, and C. G. White, Can. J. Phys. 42, 1837 (1964).

${ }_{15}$ J. H. Kusmiss and A. T. Stewart, Advan. Phys. 16 (63), 471 (1967); report, American Physical Society meeting, Washington, 1965 (unpublished).

${ }^{16}$ R. N. West, R. A. Borland, J. R. A. Cooper, and N. E. Cusack, Proc. Phys. Soc. (London) 92, 195 (1967).

${ }^{17}$ R. N. West, Low-Energy Positron Conference, Saclay, 1969 (unpublished). narrower, as is usually the case for deformed metal samples. ${ }^{18}$

Results for single crystals of tin and bismuth are shown in Figs. 3-6. Tin has not been studied earlier by this method. Single-crystal studies on bismuth have been performed by Dekhtyar and Mikhalenkov, ${ }^{19}$ but with much less resolution than in the present work. In the central paraboliclike part of the curves, the deviations from the polycrystalline result are small for both metals. Two of the bismuth curves, namely, the $[111]$ and the $[100]$ curves, are nearly equal. The peak anisotropies shown here are of the same order of magnitude as found in other nearly free-electron metals. ${ }^{1,20}$

A rather important result of these measurements is the unusually large anisotropy of the broad component of the curves - the tails-for both metals. Anisotropies of the tails of germanium and silicon curves have been

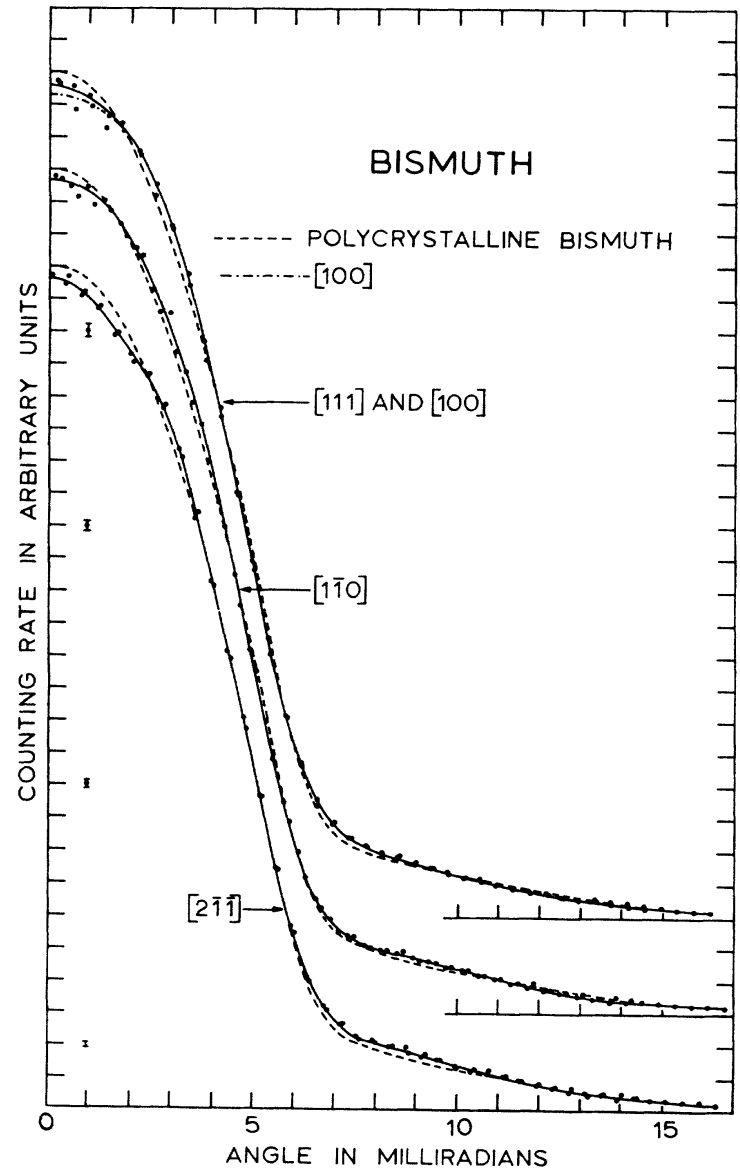

FIG. 5. Angular correlation of annihilation photons in single crystals of bismuth. The curves are normalized to the same area as the polycrystalline sample curve.

${ }^{18}$ S. Berko and J. C. Erskine, Phys. Rev. Letters 19, 307 (1967).

${ }^{19}$ I. Y. Dekhtyar and Y. S. Mikhalenkov, Dokl. Akad Nauk SSSR 136, 63 (1961) [English transl.: Soviet Phys.-Dokl. 6, 31 (1961)].

${ }_{20}$ J. J. Donaghy and A. T. Stewart, Phys. Rev. 164, 391 (1967); 


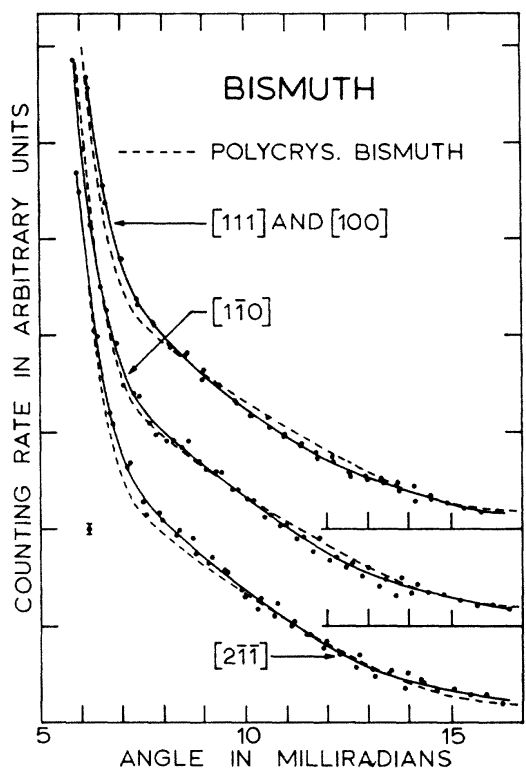

FIG. 6. Larger angle parts of the angular-correlation curves for bismuth single crystals.

reported $^{21}$; particularly, the [111] direction in germanium gave secondary peaks in the tail. Another later measurement ${ }^{7}$ of the germanium and silicon curves showed no secondary peaks. A small amount of tail anisotropy has been reported for lithium ${ }^{20}$ and copper. ${ }^{6}$ In the present study on bismuth and tin, the observed anisotropy is clearly exceeding the fluctuations due to counting statistics. In particular, the tails of the tin curve show a pronounced dependence upon crystal orientation. Tail anisotropy seems to be a rare phenomenon, although many single crystals have been investigated. A reason for this could be that only a too small part of the tail is usually measured. One could also conjecture that tail anisotropy is most pronounced in the heavier metals. Except for some rare-earth metals, ${ }^{22}$ where tails have not been investigated, single-crystal results have only been obtained for the lighter metals.

\section{DISCUSSION}

\section{A. Liquid and Deformed Samples}

According to Kusmiss and Stewart, ${ }^{1,15}$ the metals can be divided into three groups. In the first group the angular-correlation curves change upon melting. The curves for metals in the second group change below the melting point and little or no further change occurs upon melting. The third group shows no change at all. Bismuth and tin belong to the first group. This is in agreement with the data found by MacKenzie et al. ${ }^{14}$ Recently, positrons in defect metals have attracted

${ }^{21}$ P. Colombino, B. Fiscella, and L. Trossi, Nuovo Cimento 31, 950 (1964).

${ }_{22}$ R. W. Williams and A. R. Mackintosh, Phys. Rev. 168, 679 (1968). very much attention. ${ }^{23}$ It seems that only metals in the second group show appreciable dependence upon defects. Our qualitative results on deformed bismuth follow the general trend, as they show very little-if any-change in the curves because of deformation. The measured changes in the angular-correlation curves upon melting, on deformation, or upon heating below the melting point, seem qualitatively to be of the same nature. The peak becomes narrower, some smearing occurs at the peak-to-tail intersection, and the tail contribution is reduced. Figures 1 and 2 are examples of this behavior. The narrowing of the peak upon melting has recently been questioned by West. ${ }^{17}$ We think that this is clearly present in our curves; no change in the procedure of normalization can render the liquid-metal and the polycrystalline-metal peaks identical. In fact, the form of the liquid-metal central peak is triangular rather than parabolic.

In many treatments ${ }^{1,16}$ the tails of the solid- and liquid-metal curves are approximated by the same Gaussian curve, apart from a normalization factor. The curves given here-particularly the tin curves-show clearly that the tails of the liquid-metal curves are quite different from the tails of the solid-metal curves, and no Gaussian with only one adjustable constant factor could fit on both tails. It is indeed reasonable to expect that the form of the tail is changed on melting, as its anisotropy obviously must vanish above the melting point.

One noteworthy result of the angular-correlation measurements of liquid metals is that all metals, which show a change in the curves upon melting-the abovementioned first group-have rather complex crystal structures. Also, nearly all the metals with complex crystal structures which have been measured belong to the first group; selenium seems to be the only exception. The reason could be that all metals tend towards the same or nearly the same type of short-range order in the liquid phase, and that this order resembles the simpler metal structures, namely, the face-centered cubic, the body-centered cubic, and the close-packed hexagonal structures. Apart from crude qualitative models, a theory of positron annihilation on liquid

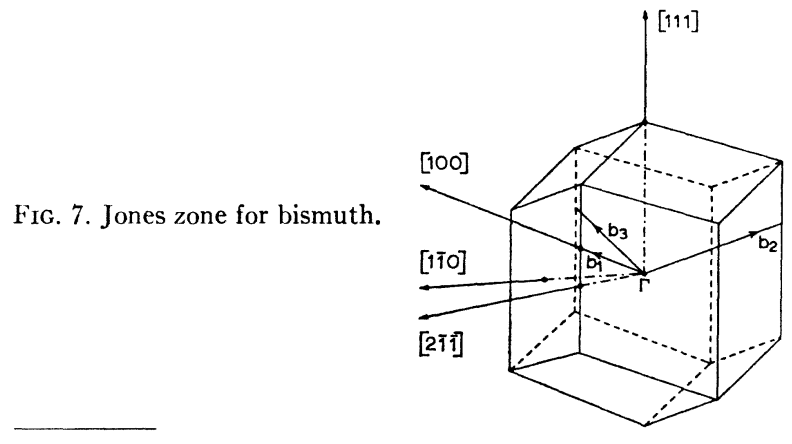

${ }^{23}$ J. C. Grosskreutz and W. E. Millett, Phys. Letters 28A, 621 (1969). 
metals does not exist today. The theoretical situation has recently been discussed by West et al..$^{16}$ and West, ${ }^{17}$ who especially tried to interpret the bismuth curves.

\section{B. Single-Crystal Samples}

At present the best way to interpret the shown single-crystal angular-correlation curves would be to apply one of the more advanced methods of bandstructure calculations, as mentioned in Sec. II. Such calculations are more difficult than the usual Fermisurface determinations, and only a few metals have been treated. ${ }^{1,24-27}$ One may anticipate much new theoretical work along these lines in the future, because such studies make possible the use of wave functions for valence electrons away from the Fermi surface, thus testing the applicability of the independent-particle model for these electrons. In the absence of sufficiently detailed theoretical calculations, we shall here only give a qualitative discussion of the measured curves in terms of more simple models.

The cross-sectional model mentioned in Sec. II is easily applied to the bismuth Jones $z^{2} e^{28}$ shown in Fig. 7 (the small pockets cannot of course be detected by positron annihilation experiments). It is seen that the [111] angular-correlation curve should be constant for angles smaller than about $3 \mathrm{mrad}$. This is not really the case, although the $[111]$ curve is rather flat for small angles. The fairly sharp turn of the [211] curve around $3 \mathrm{mrad}$ corresponds rather well to a kink in a cross-sectional area curve as function of angle for the same direction. Also, the relatively sharp transition from peak to tail in the $[1 \overline{1} 0]$ curve can be ascribed to a jump in the corresponding cross-sectional-area curve. Hence, the model predicts qualitatively some of the features of the experimental bismuth curves. The same approximation, when applied to the tin case, would only give very small corrections to the free-electron parabola, as the Fermi surface of tin is assumed to be nearly spherical. ${ }^{29}$

Another model, first proposed by Berko and Plaskett,${ }^{30}$ is the use of nearly free-electron wave functions assuming the positron wave function to be constant. In particular, this model predicts smearing of the transition between peak and tail, if Brillouin-zone boundaries, with structure factors differing from zero, are perpendicular to or nearly perpendicular to the $z$ direction and near to the Fermi surface. For tin this is the case for the [110] curve, but not for the [100] curve. The latter is, however, the curve which shows most smearing in that part of the curve where peak and tail meet. The same model predicts rather large smearing

\footnotetext{
${ }^{24}$ J. Melngailis and S. DeBenedetti, Phys. Rev. 145, 400 (1966). 25 T. L. Loucks, Phys. Rev. 144, 504 (1966).

${ }^{26}$ R. P. Gupta and T. L. Loucks, Phys. Rev. 176, 848 (1968).

${ }^{27}$ D. Stroud and H. Ehrenreich, Phys. Rev. 171, 399 (1968).

${ }^{28} \mathrm{H}$. Jones, The Theory of Brillouin Zones and Electronic States in Crystals (North-Holland Publishing Co., Amsterdam, 1962). ${ }^{29}$ G. Weisz, Phys. Rev. 149, 504 (1966).

${ }^{30}$ S. Berko and J. S. Plaskett, Phys. Rev. 112, 1877 (1958).
}

at the peak-to-tail intersection of the bismuth [110] curve. This is in contradistinction to the experimental results as mentioned above. Hence, agreement between the predicted and the experimental curves is not satisfactory for this model in the tin and bismuth case. A similar result has been found for copper by Fujiwara et al. . $^{31}$

Usually, only the structure of the peak of the angularcorrelation curves has been compared with the theoretical results. We expect that the structure and anisotropy of the tails could supply valuable additional information. A careful theoretical study of the tails observed in the present work would therefore be particularly desirable. Anisotropy of the tails of the angular-correlation curve can be explained by use of the independentparticle model (cf. Sec. II). This was especially shown by Melngailis and DeBenedetti, ${ }^{24}$ who found anisotropy in the theoretical valence-electron contribution to the tails of the lithium curves by summation over 19 different reciprocal-lattice vectors $\mathbf{g}$ in Eq. (2). Preliminary qualitative considerations indicate that the observed tail anisotropies of bismuth and tin might be calculated in the same way. On the other hand, core annihilation could also contribute so that a complete calculation would have to include the core contribution obtained by use of a realistic positron wave function. ${ }^{27}$

It is noteworthy that the lifetime of positrons in bismuth is about $18 \%$ larger than the lifetime in $\operatorname{tin},{ }^{32}$ although the density of valence electrons is nearly equal. Our data show that the angular-correlation curves are also very similar, especially if the tails are of the same relative magnitude for both metals. Hence, the lifetime difference cannot be due to core annihilation, and it must rather be caused by lattice effects. This implies that the usual electron-gas calculation methods are insufficient, and a many-body theory taking into account the ion-core potentials should be applied.

Except for these considerations, we have not found it worthwhile to pursue the interpretation of our data in terms of the simple, very approximate theories. It is obvious that the simple models are inadequate for the description of details in single-crystal angularcorrelation curves. What is needed are proper bandstructure calculations. The experimental data of this paper are presented with the hope that they may encourage such theoretical work. The present investigations are being continued by similar studies on zinc crystals.

\section{ACKNOWLEDGMENTS}

We wish to thank K. Petersen for valuable contributions to the progress of the work. Thanks are also due to members of Physics Laboratory I and III, Technical University of Denmark, for advice and help in preparing the single-crystal samples.

${ }^{31} \mathrm{~K}$. Fujiwara and O. Sueoka, J. Phys. Soc. Japan 23, 1242 (1967).

${ }^{32}$ H. Weisberg and S. Berko, Phys. Rev. 154, 249 (1967). 